\title{
OPTIMAL REPLACEMENT POLICIES FOR A DETERIORATING SYSTEM WITH IMPERFECT MAINTENANCE
}

\author{
A. RANGAN AND \\ R. ESTHER GRACE,* Indian Institute of Technology, Madras
}

\begin{abstract}
A system is repaired on failure. With probability $p$, it is returned to the 'good as new' state (perfect repair) and with probability $1-p$, it is returned to the functioning state, but is only as good as a system of age equal to its age at failure (imperfect repair). In this article, we develop replacement policies for a deteriorating system with imperfect maintenance. The successive survival times and consecutive repair times form a geometric process which is stochastically non-increasing or non-decreasing respectively. Explicit expressions are obtained for the long-run expected cost under two kinds of replacement policies based on the working age of the system and the number of imperfect repairs before a replacement.
\end{abstract}

\section{Introduction}

Most publications on maintenance policies over the last two decades have overlooked the possibility of imperfect repairs, which can be due to several limitations. Nakagawa [5] and Brown and Proschan [2], to name only two, have dealt with imperfect maintenance policies and their ramifications, although these models assume instantaneous repairs. However, for systems subject to wear and deterioration after successive repairs, the concept of cumulative damage and its effect on the survival and repair times play a dominant role. One possible way of modelling such systems with cost analysis is through the use of a non-homogeneous Poisson process [1], [6]. Lam Yeh [4] in an alternative approach has demonstrated the utility of the use of monotonic geometric processes in such studies. Our endeavour in this note is to use Lam Yeh's approach to consider the optimal replacement policies for a deteriorating system in which one of the two kinds of repair, a perfect repair or an imperfect repair, is performed at each of the repair times.

\section{The model}

We consider a system which is put in operation at $t=0$. Every time a failure occurs, the system is repaired so that the repaired unit is as good as new with probability $p$ (perfect repair), while with probability $q=1-p$, it is restored to its condition just prior to failure (imperfect repair). If $X_{n}$ is the survival time after the $(n-1)$ th successive minimal repair, then $\left\{X_{n} ; n=1,2, \cdots\right\}$ forms a geometric process with parameter $a \geqq 1$ and $E\left[X_{1}\right]=\lambda>0$ (refer to Lam Yeh [4] for details). The repairs take a non-negligible amount of time so that if $Y_{n}$ is the repair time (perfect or imperfect) after the $n$th failure (the previous $(n-1)$ being imperfect repairs), then $\left\{Y_{n} ; n=1,2, \cdots\right\}$ constitute a geometric process with parameter $b$, $(0<b \leqq 1)$ and $E\left[Y_{1}\right]=\mu>0$. We further assume that $\left\{X_{n}\right\}$ and $\left\{Y_{n}\right\}$ are independent.

Two kinds of replacement policy for the system are considered:

(i) A replacement policy $T$ : The system is replaced with a new one whenever the working age of the system reaches $T$.

Received 2 February 1989; revision received 5 June 1989.

${ }^{*}$ Postal address for both authors: Department of Mathematics, Indian Institute of Technology, Madras - 600 036, India. 
(ii) A replacement policy $N$ : The system is replaced at the time of completion of the $N$ th successive imperfect repair since the last replacement.

Under such policies we observe that the system renews itself at each of the instants of replacement or perfect repairs, whichever is earlier. Also in running the system one encounters the following costs:

$$
\begin{aligned}
& C_{1}: \text { cost/unit repair time of a perfect repair } \\
& C_{2}: \text { cost/unit repair time of an imperfect repair } \\
& C_{3}: \text { cost/replacement } \\
& \text { Y: reward/unit of working time. }
\end{aligned}
$$

\section{Replacement policy $T$}

Under this policy the length of a renewal cycle $L$ is

$$
L= \begin{cases}T+V_{k} & \begin{array}{l}
\text { if there is no perfect repair until the } \\
\text { working age of the system reaches } T, \\
U_{k}<T \leqq U_{k+1} ; k=0,1,2, \cdots
\end{array} \\
U_{k}+V_{k} & \begin{array}{l}
\text { if the } k \text { th repair is a perfect one and } \\
U_{k}<T ; k=1,2, \cdots
\end{array}\end{cases}
$$

where $U_{n}=\sum_{i=1}^{n} X_{i}$ and $V_{n}=\sum_{i=1}^{n} Y_{i}$ with $U_{0}=0$ and $V_{0}=0$. Thus $L_{1}(T)$, the expected duration of a renewal cycle, is given by

$$
L_{1}(T)=E[L]=E\left[\left(T+\sum_{i=1}^{k} Y_{i}\right) I\left(U_{k}<T<U_{k+1}\right)\right]+E\left[\sum_{i=1}^{k}\left(X_{i}+Y_{i}\right) I\left(U_{k}<T\right)\right]
$$

where $I(\cdot)$ is the indicator function. After simplification, $L_{1}(T)$ reduces to

$$
L_{1}(T)=T+\sum_{n=1}^{\infty} \frac{\mu}{b^{n-1}} q^{n-1} F_{n}(T-0)+\sum_{n=1}^{\infty} q^{n-1} p\left[\Gamma_{n}(T)+\sum_{k=1}^{n} \frac{\mu}{b^{k-1}} F_{n}(T-0)\right]
$$

where $F_{n}(\cdot)$ is the distribution function of $U_{n}, \Gamma_{n}(T)=\int_{0}^{T} \bar{F}_{n}(u) d u$ and we have made use of the facts that $E\left[Y_{n}\right]=\mu / b^{n-1}$ and $\left\{X_{n}\right\}$ and $\left\{Y_{n}\right\}$ are independent. Also using (1), $D(T)$ the expected cost per renewal cycle is given by

$$
\begin{aligned}
D(T)= & \sum_{n=1}^{\infty} q^{n-1}\left(C_{3}+C_{2} \sum_{k=1}^{n} \frac{\mu}{b^{k-1}}-r T\right)\left(F_{n}(T)-q F_{n+1}(T)\right) \\
& +\sum_{n=1}^{\infty} q^{n-1} p\left[\left(C_{1} \frac{\mu}{b^{n-1}}+C_{2} \sum_{k=1}^{n-1} \frac{\mu}{b^{k-1}}\right) F_{n}(T-0)-r \Gamma_{n}(T)\right] \\
& +\left(C_{3}-r T\right) \bar{F}_{i}(T) .
\end{aligned}
$$

From standard renewal theory the long-run expected cost per unit time is given by

$$
C_{1}(T)=\frac{D(T)}{L_{1}(T)}=\frac{\text { the expected cost } / \text { renewal cycle }}{\text { the expected length of a renewal cycle }} .
$$

Optimal $T^{*}$ is obtained from (5) using analytical or numerical methods.

\section{Replacement policy $N$}

Under this policy the long-run expected cost per unit time $C_{2}(N)$ is given by

$$
C_{2}(N)=\frac{D(N)}{L(N)}
$$


where

$$
\begin{aligned}
D(N)= & \left(C_{3}+C_{2} \sum_{k=1}^{N-1} \frac{\mu}{b^{k-1}}\right) q^{N-1} \\
& +\sum_{k=2}^{N-1} q^{k-1} p\left(C_{1} \frac{\mu}{b^{k-1}}+C_{2} \sum_{j=1}^{k-1} \frac{\mu}{b^{j-1}}\right) \\
& -r\left(q^{N-1} \sum_{j=1}^{N-1} \frac{\lambda}{a^{j-1}}+\sum_{k=2}^{N-1} q^{k-1} p \sum_{j=1}^{k-1} \frac{\lambda}{a^{j-1}}\right)
\end{aligned}
$$

and

$$
L(N)=\sum_{k=1}^{N-1}\left(\frac{\mu}{b^{k-1}}+\frac{\lambda}{a^{k-1}}\right) q^{N-1}+\sum_{k=2}^{N-1} q^{k-1} p \sum_{j=1}^{k-1}\left(\frac{\mu}{b^{j-1}}+\frac{\lambda}{a^{j-1}}\right) .
$$

We wish to observe that in the case when $p=0$ so that every repair on failure is an imperfect repair, the cost equations (2)-(8) reduce to the ones obtained by Lam Yeh [5] (see his Equations (4) and (6)). The situation when $\mu=0$ (in the limit $\mu \rightarrow 0$ ), so that the repair time is negligible and $a=1$ is the case that has been dealt with by Fontenot and Proschan [3]. Since the repair is assumed to be instantaneous, they have assumed a cost of $C_{1}$ per minimal repair instead of $C_{1}$ per unit repair time as in our case and which can be deduced from ours. The further case of $p=0$ results in a non-homogeneous Poisson process with intensity function $r(t)$ corresponding to $F(t)$.

\section{Acknowledgement}

The authors wish to thank the referee for his suggestions, in the light of which the earlier draft of the paper was greatly condensed.

\section{References}

[1] ABDel-Hameed, M. (1986) Optimum replacement of a system subject to shocks. J. Appl. Prob. 23, 107-114.

[2] Brown, M. and Proschan, F. (1983) Imperfect repair J. Appl. Prob. 20, 851-859.

[3] Fontenot, R A. ANd Proschan, F. (1984) Some imperfect maintenance models. In Reliability Theory and Models, ed. M. Abdel-Hameed, Erhan Çinlar and Joseph Quinn. Academic Press, London.

[4] Lam Yeh (1988) A note on the optimal replacement problem. Adv. Appl. Prob. 20, 479-482.

[5] NAKagawa, T. (1980) A summary of imperfect PM Policies with minimal repair RAIRO Rech. Operat. 14, 249-255.

[6] Rangan, A. AND Esther Grace, R. (1988) A non-Markov model for the optimum replacement of self-repairing systems subject to shocks. J. Appl. Prob. 25, 375-382. 